\title{
Tipificación de Rhamnus lotus L. (Rhamnaceae)
}

\author{
Pedro Pablo Ferrer-Gallego \& Emilio Laguna
}

Servicio de Espacios Naturales y Biodiversidad. Centro para la Investigación y Experimentación Forestal (CIEF). Generalitat Valenciana. Av. Comarques del País Valencià, 114, E-46930, Quart de Poblet, Valencia.

\section{Correspondencia}

P.P. Ferrer-Gallego

E-mail: flora.cief@gva.es

Recibido: 12 noviembre 2012

Aceptado: 14 febrero 2013

Publicado on-line: 18 febrero 2013

\section{Resumen}

Se lectotipifica Rhamnus lotus L. [Ziziphus lotus (L.) Lam.] (Rhamnaceae), para lo cual se ha estudiado todo el material depositado en los diferentes herbarios donde se conserva material de Linneo y la información bibliográfica del protólogo. Se propone como lectótipo el material conservado en el herbario de Linneo (LINN)

Palabras clave: Lectótipo, Linneo, Nomenclatura, Rhamnaceae.

\begin{abstract}
Typification of Rhamnus lotus L. (Rhamnaceae)

A lectotypification of Rhamnus lotus L. [Ziziphus lotus (L.) Lam.] (Rhamnaceae) is proposed, based on the study of all the material deposited in several herbaria where Linnaean specimens are preserved and the bibliographic information obtained in the protologue. The material preserved in the Linnean Herbarium (LINN) is proposed as the lectotype.
\end{abstract}

Key words: Lectotype, Linneo, Nomenclature, Rhamnaceae.

\section{Introducción}

En el género Rhamnus L. (Rhamnaceae) hay todavía muchos nombres aún no tipificados. En este trabajo se lectotipifica el nombre de Linneo Rhamnus lotus que, en la actualidad, se combina en el género Ziziphus Mill.-Ziziphus lotus (L.) Lam., Encycl. 3(1): 317 (1789). Se trata de un arbusto fuertemente espinoso de 1-3 metros de altura, con ramas zigzagueantes, hojas alternas, ovadas o elípticas y nervios bien marcados. El fruto es una drupa (nuculanio) globosa de color pardorojizo, comestible. Se distribuye principalmente por la cuenca sur del Mediterráneo, por el norte de África desde el Magreb a Egipto, por el sureste de España peninsular, Sicilia y Grecia, y avanzando al este por Israel, Jordania y Arabia. Esta especie tiene multitud de utilidades a lo largo de todo el territorio por donde se reparte, siendo una planta de gran importancia para la agricultura y la alimentación, pero también empleada con fines me- dicinales en muchas sociedades.

Tras la búsqueda de posible material tipo, hemos localizado, en el herbario de Linneo on line (LINN), un pliego con material (LINN 262.27) que contiene un fragmento en buen estado de conservación, aunque sin flores ni frutos. En la parte inferior de la cartulina, se lee manuscrito por Linneo el número de orden y el epíteto específico de la especie en el Species Plantarum: "6. Lotus". Además de este espécimen, también constituye material tipificable y original el icon "632. $\mathrm{Zi}$ ziphus Sylvestris I.R.H. 627.-Seedra Arabum, quae et Lotus Veterum" de Shaw (1738: 47, f. 632) (Fig. 1), citado en el protólogo con el nombre de "Zizyphus sylvestris". En el protólogo de Linneo aparece indicado el número 631 para hacer referencia al grabado de Ziziphus sylvestris de Shaw, cuando en la obra de este autor aparece con el número 632 (SHAW, 1738: 47, f. 632), lo que parece indicar que se trata de una errata de Linneo. 


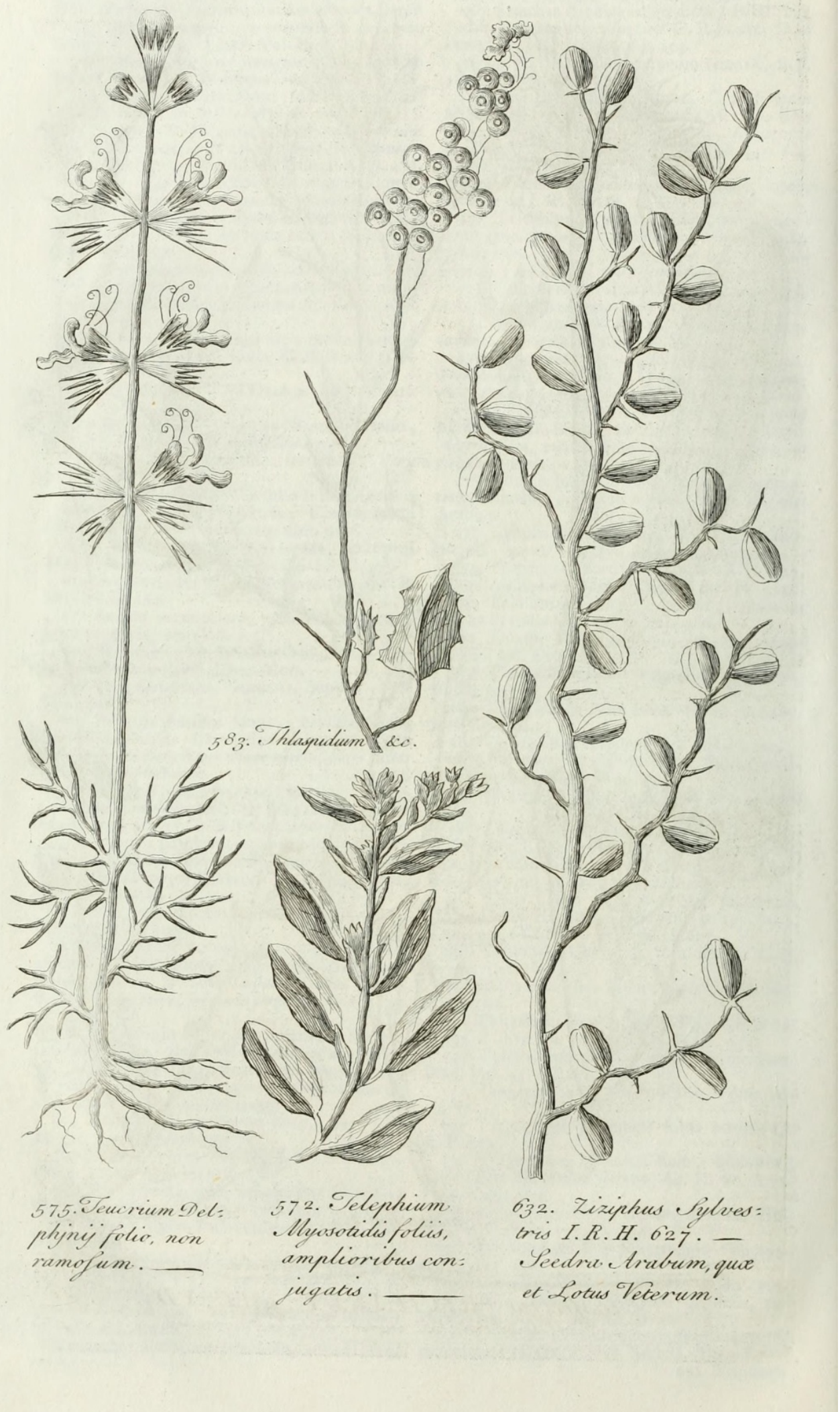

Figura 1. Rhamnus lotus L., [icon] en SHAW, Cat. Pl. Afr. As.: 47, f. 632 (1738).

Figure 1. Rhamnus lotus L., [icon] in SHAW, Cat. Pl. Afr. As.: 47, f. 632 (1738). 
Además, hay en el herbario S-LINN tres pliegos con materiales de la especie, pero por las anotaciones manuscritas que contienen parecen posteriores a la redacción del Species Plantarum. El pliego Herb. Linn. No. 95.15 (S-LINN) fue recibido por el hijo de Linneo de Anders Dahl, contiene un fragmento y en el reverso de la hoja aparece escrito "Rhamnus lotus//Dahl a Linne f.". El pliego Herb. Linn. No. 95.17 (S-LINN) conserva dos fragmentos y en el anverso de la hoja aparece escrito "Rhamnus 6 Lotus/Sp. Pl. 194/ = Zizyphus Lotus Lam." y "Zizyphus/Lotus Lam.", mientras que en el reverso se reproduce la frase diagnostica de Linneo "Rhamnus aculeis geminatis altero recurvo, foliis ovato-oblongis / Linn. Spec. plant. 194. 6.". Por último, el pliego Herb. Linn. No. 95.19 (S-LINN), también contiene un fragmento de esta especie con las siguientes indicaciones en el anverso del pliego "Rh. Lotus L./Ziz. Lotus Lam./(specim. juvenile)." "Sp. Pl. 195//11// Spina Christi.", y escrito en la parte superior del reverso de la hoja "Rhamnus aculeis geminatis rectis, foliis ovatis/Linn. Spec. pl. 194. 11." y en la inferior "Spina Christi//16 Zizyphus Spina Christi//Rhamnus Spina Christi Linn./ Hort.".

Según el Art. 9.10 del ICBN (McNeill \& al., 2006), los especímenes, si existen, tienen prioridad para ser elegidos como lectótipos frente a las ilustraciones "9.10. In lectotype designation, an isotype must be chosen if such exists, or otherwise a syntype if such exists. If no isotype, syntype or isosyntype (duplicate of syntype) is extant, the lectotype must be chosen from among the paratypes if such exist. If no cited specimens exist, the lectotype must be chosen from among the uncited specimens and cited and uncited illustrations which comprise the remaining original material, if such exist". Por lo tanto, se considera como el mejor candidato a lectótipo del nombre de Linneo Rhamnus lotus el pliego conservado en LINN. Se propone, por lo tanto, lo siguiente:

\section{Tipificación}

Rhamnus lotus L., Sp. Pl. 1: 194 (1753)

$\equiv$ Ziziphus lotus (L.) Lam., Encycl. 3(1): 317 (1789)

Ind. loc.: "Habitat in Regno Tunetano"

Type: Lectotypus (hic designatus): Herb. Linn. No. 262.27 (LINN).

\section{Agradecimientos}

Al Dr. Alberto Herrero Nieto (Real Jardín Botánico de Madrid) por sus valiosos comentarios y la revisión general del manuscrito.

\section{Referencias}

LINN. The Linnean Collections. LINN 262.27 Rhamnus lotus (Herb Linn). Disponible en http://www.linneanonline.org/2749/ (accedido el 1 de noviermbre de 2012).

McNeill J, Barrie FR, Burdet HM, Demoulin V, Hawksworth DL, Marhold K, Nicolson DH, Prado J, Silva PC, Skog JE, Wiersema JH \& Turland NJ. (eds.). 2006. International Code of Botanical Nomenclature (Vienna Code) adopted by the Seventeenth International Botanical Congress Vienna, Austria, July 2005. Regnum Vegetabile 146. A.R.G. Gantner Verlag, Ruggell, Liechtenstein.

Shaw T. 1738. Catalogus plantarum quas in variis Africae \& Asiae partibus. Oxford. 